\title{
Precision physics with a wide band super neutrino beam
}

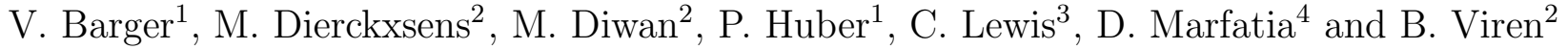 \\ ${ }^{1}$ Department of Physics, University of Wisconsin, Madison, WI 53706 \\ ${ }^{2}$ Brookhaven National Laboratory, Upton, NY 11973 \\ ${ }^{3}$ Department of Physics, Columbia University, New York, NY 10027 \\ ${ }^{4}$ Department of Physics and Astronomy, University of Kansas, Lawrence, KS 66045
}

\begin{abstract}
We carry out a state-of-the-art assessment of long baseline neutrino oscillation experiments with wide band beams. We describe the feasibility of an experimental program using existing high energy accelerator facilities, a new intense wide band neutrino beam $(0-6 \mathrm{GeV})$ and a proposed large detector in a deep underground laboratory. We find that a decade-long program with $1 \mathrm{MW}$ operation in the neutrino mode and $2 \mathrm{MW}$ operation in the antineutrino mode, a baseline as long as the distance between Fermilab and the Homestake mine $(1300 \mathrm{~km})$ or the Henderson mine $(1500 \mathrm{~km})$, and a water Cherenkov detector with fiducial mass $\sim 300 \mathrm{kT}$ has optimum sensitivity to a nonzero $\theta_{13}$, the mass hierarchy and to neutrino $\mathrm{CP}$ violation at the $3 \sigma$ C. L. for $\sin ^{2} 2 \theta_{13}>0.008$. This program is capable of breaking the eight-fold degeneracy down to the octant degeneracy without additional external input.
\end{abstract}




\section{Introduction}

There is abundant evidence that neutrinos oscillate and hence have mass [1]. Atmospheric neutrinos oscillate with a mass-squared difference $\left|\Delta m_{31}^{2}\right| \sim 0.0025 \mathrm{eV}^{2}$ and mixing angle $\theta_{23} \sim \pi / 4$ [2] while the corresponding parameters for solar neutrinos are $\Delta m_{21}^{2} \sim 8 \times 10^{-5} \mathrm{eV}^{2}$ and $\theta_{12} \sim \pi / 6[3]$. The unknowns are the angle $\theta_{13}$ coupling the atmospheric and solar sectors, the CP-violating phase $\delta_{\mathrm{CP}}$, and the sign of $\Delta m_{31}^{2}$, which fixes the hierarchy of neutrino masses. All we presently know about $\theta_{13}$ is that $\sin ^{2} \theta_{13}<0.05$ at the $2 \sigma$ C. L. [4. Measurements of the unknown parameters are the main goals of future long-baseline neutrino experiments. Complete and precise experimental knowledge of neutrino parameters is essential to test neutrino mass models. Moreover, a determination of the type of mass hierarchy is of importance for the feasibility of observing neutrinoless double beta if the heaviest neutrino is $\mathcal{O}(0.05) \mathrm{eV}$. An observation of this decay will provide much confidence in the Majorana nature of neutrinos.

In the near future, experiments with low energy conventional neutrino beams will attempt to detect $\nu_{\mu} \rightarrow \nu_{e}$ transitions at the atmospheric scale. Concurrently, reactor experiments will try to determine or constrain $\theta_{13}$ by observing $\bar{\nu}_{e}$ disappearance at the atmospheric scale. However, the complementary information gathered from both types of experiments will be inadequate to determine the mass hierarchy or to establish CP-violation in the lepton sector. In recognition of this fact, planning is underway for future facilities that will address these outstanding issues.

Two types of neutrino beams are being considered for future long-baseline experiments: off-axis beams with narrow band energy spectra (as will be used in the Tokai-to-Kamioka (T2K) experiment at Japan Proton Accelerator Proton Complex (J-PARC) [5], and proposed for the NuMI Off-axis $\nu_{e}$ Appearance Experiment or $\mathrm{NO} \nu \mathrm{A}$ [ 6]) and a wide band beam [7, 8] for which the neutrino energy is inferred from quasielastic scattering events.

Off-axis beams 9] are advocated because their beam energy is narrow and depend to first order only on the kinematics of pion decay, so that a counting experiment can be carried out at an oscillation maximum. Another positive feature of the narrow beam is the reduction of a high energy tail which could contribute background to reconstructed events at low energy via neutral-current feed down. The features that make off-axis beams attractive also lead to their limitations. The very nature of a counting experiment precludes the possibility of using spectral energy information. Consequently, it is virtually impossible to resolve the eight-fold parameter degeneracy [10] (which arises from three independent two-fold degeneracies $\left(\delta_{\mathrm{CP}}, \theta_{13}\right), \operatorname{sgn}\left(\Delta m_{31}^{2}\right)$ and $\left(\theta_{23}, \pi / 2-\theta_{23}\right)$ [10, 11] $)$, unless multiple measurements with separate detectors are made at different off-axis angles and/or baselines. The capabilities and optimization of experiments with off-axis beams have been studied extensively [12.

Wide band beams possess the advantages of a higher on-axis flux and a broad energy spectrum. In an idealized sense, one may think of them as enabling many off-axis experiments in a single 
experiment. Thus, parameter degeneracies may be resolved in a single wide band beam experiment. The disadvantage of a wide band beam is the need to determine the neutrino energy with good resolution and eliminate background from high energy tails of the spectrum. Therefore, detectors with good energy resolution and neutral-current rejection are needed to use the broad spectrum fruitfully and to reject the feed down from the high-energy tail efficiently.

The possibility of working with large detectors may not be too far into the future [13, 14, 15]. Two concrete proposals are under consideration for a Deep Underground Science and Engineering Laboratory (DUSEL) [16] in the U.S., one at the Homestake mine in South Dakota, the other at the Henderson mine in Colorado. DUSEL will be designed to accomodate Mton class multipurpose detectors. The physics program of these detectors will include nucleon decay, supernova neutrino detection, as well as long-baseline neutrino oscillation physics with an accelerator beam.

The prospects of a very long-baseline experiment consisting of a wide band super neutrino beam and a large water Cherenkov detector were analysed in Refs. [8, 17], where, in the spirit of a first analysis, correlations and degeneracies were only partially accounted for. Preliminary work had suggested that sufficient background suppression could be achieved so as to extract the quasielastic events with sufficient purity. Since then, further detailed assessments with a full simulation of a water Cherenkov detector have been performed [18. The main result of that work is that neutral current backgrounds can be sufficiently suppressed while maintaining good signal efficiency with new software techniques. The remaining signal events are dominated by quasielastics, but have significant contamination of other $\nu_{e}$ induced charged current events. Nevertheless, neutrino energy resolution in the range of $\sim 10 \%$ can be maintained. This work will be reported elsewhere in detail.

In this paper, we perform a thorough state-of-the-art analysis with a realistic treatment of systematic errors, correlations and degeneracies so as to define an optimum program using a wide band beam. We consider accelerator and detector requirements for a feasible wide band experiment in the next two sections. In Section 4 we discuss our analysis methodology and present results of the analysis in Section 5. We summarize in Section 6.

\section{Accelerator Requirements}

A number of recent studies have examined possible intensities and neutrino spectra from U.S. proton accelerators [19, 20, 21]. Here we summarize the understanding of the intensity versus proton energy available in the U.S. currently, in the near future, and with upgrades.

High energy multi-MW proton beams are under consideration at FNAL. An examination is underway to increase the total power from the $120 \mathrm{GeV}$ Main Injector (MI) complex after the Tevatron program ends 22. In this scheme protons from the $8 \mathrm{GeV}$ booster, operating at $15 \mathrm{~Hz}$, will be stored in the antiproton accumulator (which becomes available after the shutdown of the Tevatron program) while the MI completes its acceleration cycle. Combining the techniques of 
momentum stacking using the antiproton accumulator and slip-stacking using the recycler will raise the total intensity in the MI to $\gtrsim 1 \mathrm{MW}$ at $120 \mathrm{GeV}$. In the ideal case, the length of the acceleration cycle is proportional to the proton energy, making the average beam power proportional to the final proton energy. However, there are fixed time intervals in the beginning and the end of the acceleration cycle for stable operation. These become important at low energies and reduce the performance below the ideal. Current projections suggest that $\sim 0.5 \mathrm{MW}$ operation between $40-60 \mathrm{GeV}$ and $\gtrsim 1 \mathrm{MW}$ operation at $120 \mathrm{GeV}$ is possible.

Ambitious plans at FNAL call for a $8 \mathrm{GeV}$ super-conducting LINAC that can provide $1.5 \times 10^{14}$ $H^{-}$ions at $10 \mathrm{~Hz}$ corresponding to $2 \mathrm{MW}$ of total beam power [19]. Some of the $8 \mathrm{GeV}$ ions could be injected into the MI to provide high proton beam power, 1 to $2 \mathrm{MW}$, at energy between 40 and $120 \mathrm{GeV}$; e.g., $40 \mathrm{GeV}$ at $\sim 2 \mathrm{~Hz}$ or $120 \mathrm{GeV}$ at $\sim 0.67 \mathrm{~Hz}$. Such a plan allows for flexibility in the choice of proton energy for neutrino production.

The BNL Alternating Gradient Synchrotron (AGS) operating at $28 \mathrm{GeV}$ currently can provide about $1 / 6 \mathrm{MW}$ of beam power. This corresponds to an intensity of about $7 \times 10^{13}$ protons in a 2.5 microsecond pulse every 2 seconds. The AGS complex can be upgraded to provide a total proton beam power of $1 \mathrm{MW}$ [21]. The main components of the accelerator upgrade at BNL are a new 1.2 GeV Superconducting LINAC to provide protons to the existing AGS, and new magnet power supplies to increase the ramp rate of the AGS magnetic field from about $0.5 \mathrm{~Hz}$ to $2.5 \mathrm{~Hz}$. For 1 MW operation the protons from the accelerator will be delivered in pulses of $9 \times 10^{13}$ protons at 2.5 $\mathrm{Hz}$. It has been determined that $2 \mathrm{MW}$ operation of the AGS is also possible by further upgrading the synchrotron to $5 \mathrm{~Hz}$ repetition rate and with further modifications to the LINAC and the RF systems. A new neutrino beam could be built with conventional horn focussed technology and a $200 \mathrm{~m}$ long pion decay tunnel placed on the slope of a specially built hill. Such a beam could be aimed at a detector $\sim 2500 \mathrm{~km}$ away.

To observe multiple oscillation nodes in the $\nu_{\mu}$ disappearance channel, it is necessary to have a wide band beam with energies from 0 to $6 \mathrm{GeV}$. Protons with energy above $\sim 20 \mathrm{GeV}$ are needed to provide such a flux, which is clearly possible at both FNAL and BNL. The spectrum that is used in this paper was obtained using $28 \mathrm{GeV}$ protons and a 200 meter long decay tunnel. For details of this spectrum see Refs. [17, 23]. Recent calculations have shown that similar, but more intense spectra per unit beam power can be obtained using 40 or $60 \mathrm{GeV}$ protons from FNAL, where the meson decay tunnel could be made longer than 200 meters [24].

In our analysis we assume that the spectrum from either the FNAL or the BNL beam will be the same with a total average beam power of $1 \mathrm{MW}$. This allows us to make a proper comparison of the physics issues regarding the baselines. We comment on the additional flexibility obtained by the ability to change the proton energy at FNAL as well as the longer decay tunnel. We also comment on the impact on the sensitivity with $0.5 \mathrm{MW}$ of average power from FNAL. It should be noted that 0.5 MW operation from FNAL does not appear to require a major upgrade. 
If a large detector [13, 14, 15] is located at Homestake (HS) or Henderson (HD), the beam from FNAL (BNL) will have to traverse a distance of $1290 \mathrm{~km}$ or $1495 \mathrm{~km}(2540 \mathrm{~km}$ or $2770 \mathrm{~km})$, respectively. At FNAL the inclination will be about $5.8^{\circ}$ (HS) or $6.7^{\circ}$ (HD). The existing experience at FNAL, from building the NuMI beam, could be extended to build a new beam to HS or HD. At BNL the beam would have to be built at an inclination of about $11.4^{\circ}$ (HS) or $12.4^{\circ}$ (HD). Current design for such a beam requires the construction of a hill with a height of about $50 \mathrm{~m} \mathrm{[21].}$ Such a hill will have the proton target at the top of the hill and a $200 \mathrm{~m}$ long decay tunnel on the downslope. In either case, it is adequate to have a decay tunnel with length shorter (200 to $400 \mathrm{~m}$ ) than the NuMI tunnel $(750 \mathrm{~m})$ to obtain the needed flux. Since the focus is on lower energies, the beam intensity is maintained if the decay tunnel can be made about $4 \mathrm{~m}$ in diameter.

Another advantage of the wide tunnel is that the option of running with a narrow band beam using the off-axis technique could be preserved. A $4 \mathrm{~m}$ diameter tunnel could permit the rotation of the target and horn assembly so that a $1^{\circ}$ off-axis beam could be sent to the far detector.

\section{Detector Requirements}

A water Cherenkov detector with $300 \mathrm{kT}$ fiducial mass can be built in the same manner as the SuperKamiokande detector (with 20 inch photo-multipliers placed on the inside detector surface covering approximately $40 \%$ of the total area) 25] by simply scaling it to larger size or by building several detector modules [13, 14]. Such a detector placed underground at DUSEL could have a low energy threshold ( $\lesssim 10 \mathrm{MeV}$ ), good energy resolution $(\sim 10 \%)$ for single particles, good muon/electron separation ( $\lesssim 1 \%$ ), and time resolution ( $\lesssim$ few ns). As noted earlier, it is important to obtain good energy resolution when using a wide band beam. This can be achieved in a water Cherenkov detector by separating quasielastic scattering events with well identified leptons in the final state from the rest of the charged-current (CC) events. The fraction of quasielastics in the total CC rate with the spectrum used in this paper is about $23 \%$ for the neutrino beam and $39 \%$ for the antineutrino beam.

Separation of quasielastic events from the CC and neutral-current (NC) background is being used in the $\mathrm{K} 2 \mathrm{~K}$ experiment [26]. For the program considered here an essential problem is to separate electron shower events from other NC events, especially events containing a single $\pi^{0}$ in the final state. The goal is to search for $\nu_{e}$ induced showering events in the 0.5 to $4 \mathrm{GeV}$ range. Single $\pi^{0}$ particles with energies of $1,2,3$ and $4 \mathrm{GeV}$ decay to two photons with a minimum and most probable opening angle of $16,8,5$, and 4 degrees, respectively. The probability of a decay with an opening angle of more than $20^{\circ}$ for $1,2,3$ and $4 \mathrm{GeV} \pi^{0}$ 's is $40 \%, 8.2 \%, 3.6 \%$, and $2.0 \%$, respectively. In a water Cherenkov detector the position where the $\pi^{0}$ photons convert cannot be measured with sufficient precision from the pattern of Cherenkov light which tends to be two overlapping showering rings. At low $\pi^{0}$ energies the opening angle is sufficiently large 
compared to the Cherenkov angle $\left(42^{\circ}\right)$ that single $\pi^{0}$ 's can be separated quite effectively. At energies greater than $2 \mathrm{GeV}$, however, the small angular separation between the two photons makes such separation difficult. It is well known that resonant single pion production in neutrino reactions has a rapidly falling cross section as a function of momentum transfer, $q^{2}$, up to the kinematically allowed value [27]. This characteristic alone suppresses the background by more than 2 orders of magnitude for $\pi^{0}$ (or shower) energies above $2 \mathrm{GeV}$ [28]. Therefore a modest $\pi^{0}$ background suppression (by a factor of $\sim 15$ below $2 \mathrm{GeV}$ and $\sim 2$ above $2 \mathrm{GeV}$ ) makes the $\pi^{0}$ background manageable level over the entire spectrum. Such background suppression has recently been demonstrated using complete simulation and reconstruction in Ref. [18].

It has been argued that a liquid Argon time projection chamber (LARTPC) could be built with total mass approaching $100 \mathrm{kT}$ [15]. A fine grained detector such as this has excellent resolution for separating tracks, making it possible to use a large fraction of the $\mathrm{CC}$ events (rather than only the quasielastic events) to determine the neutrino energy spectrum. A LARTPC also has much better particle identification capability. Consequently, a LARTPC with a total fiducial mass of $\sim 100 \mathrm{kT}$ is expected to have similar performance as a $300 \mathrm{kT}$ water Cherenkov detector.

We assume a detector performance based on Ref. [18. For the physics sensitivities we assume $1 \mathrm{MW}$ operation for 5 years (at $1.7 \times 10^{7} \mathrm{sec} / \mathrm{yr}$ ) in the neutrino mode, $2 \mathrm{MW}$ operation for 5 years in the antineutrino mode and a detector fiducial mass of $300 \mathrm{kT}$. With the running times, the accelerator power level, and the detector mass fixed, we consider baselines in the range of 500 to $3000 \mathrm{~km}$. With $1 \mathrm{MW}$ of a $28 \mathrm{GeV}$ proton beam, a baseline of $1300 \mathrm{~km}$, and a 300kT fiducial volume detector we calculate $\sim 230000$ muon charged current and $\sim 77000$ neutral current events in 5 years of running in the neutrino mode in the absence of oscillations. Under the same running conditions in the antineutrino mode (with the horn current reversed) we find a total of $\sim 74000$ antimuon charged current and $\sim 27000$ neutral current events; approximately $20 \%$ of the event rate in the antineutrino beam will be due to wrong-sign neutrino interactions. For both neutrino and antineutrino running, approximately $\sim 0.7 \%$ of the CC rate will be from electron CC events which form a background to the $\nu_{\mu} \rightarrow \nu_{e}$ search. As an example, the total number of electron neutrino appearance events and the expected background for $\sin ^{2} 2 \theta_{13}=0.1$ and a normal hierarchy as a function of baseline are shown in Fig. [1,

The event rates for other beam configurations using 40 or $60 \mathrm{GeV}$ proton beam have been calculated in Ref. 24]. For equal proton beam power, higher proton energy could result in as much as a $\sim 50 \%$ increase in the total event rate with a concomitant increase in the background. A careful evaluation of the optimization of event rate versus background could reduce the running time by a significant factor. Preliminary calculations suggest that the physics sensitivity using other beam configurations will remain approximately the same as the calculations reported in this paper after accounting for the effects of spectral variations on the backgrounds and signal. 


\section{Analysis techniques}

We simulate the expected event counts for the experimental program described above and compute physics sensitivities using the GLoBES software package 29]. The description of the detector performance follows from the results in Ref. [18]. We used this detector performance to create large numbers of Monte Carlo events for the following set of event classes, which in turn, are used to create migration matrices suitable for GLoBES:

- $\nu_{e}$ appearance signal

- $\nu_{\mu}$ disappearance signal

- $\nu_{e}$ beam events

- NC background mainly consisting of single $\pi^{0}$ production.

- Other NC events.

- CC events with additional undetected particles.

We have a set of six matrices for neutrinos and antineutrinos each. The background of antineutrinos in the neutrino beam is very small since it is suppressed both in production and detection by the cross section ratio of neutrinos to antineutrinos. For antineutrino running the opposite is true. There the neutrino (wrong-sign) contamination in the beam is nonnegligible due to the enhancement by the cross section ratio. Therefore, we account for this background via 6 additional matrices that correspond to the ones above for treating the neutrinos in the antineutrino beam. Our calculations show that the background due to tau neutrino interactions is low and can be safely ignored because the spectrum we use has low energy. When combined with the high threshold $(3.5 \mathrm{GeV})$ for tau production as well as the low $\nu_{\tau} \mathrm{CC}$ cross section, the background is not significant. If higher energy protons are used to create a spectrum with a high energy tail, then this background must be included. We have carefully checked that the GLoBES calculation of event rates agrees with the direct result of the Monte Carlo for the case of no oscillations.

Once event rates are computed, the next step is the calculation of a $\chi^{2}$ function and the inclusion of systematic errors. We use a $\chi^{2}$ function for Poissonian processes as given, e.g., in Ref. [30. Systematic errors are implemented using a pull approach as in Ref. [31. The systematics considered are a normalization error for each type of signal, i.e., $\nu_{e}$ appearance, $\bar{\nu}_{e}$ appearance, $\nu_{\mu}$ disappearance and $\bar{\nu}_{\mu}$ disappearance of $1 \%$ each, uncorrelated between the four types of signal. For the sum of all backgrounds to each signal we assume a $10 \%$ uncertainty in the normalization, again uncorrelated between the four types of signal. 
As input or true values for the oscillation parameters and the Gaussian $1 \sigma$ ranges (in anticipation of precision measurements from near future experiments) we use:

$$
\begin{array}{rlrl}
\theta_{12} & =0.55 \pm 10 \%, & \Delta m_{21}^{2} & =(8.0 \pm 0.8) \cdot 10^{-5} \mathrm{eV}^{2}, \\
\theta_{23} & =\pi / 4 \pm 5 \%, & \Delta m_{31}^{2}=(2.5 \pm 0.125) \cdot 10^{-3} \mathrm{eV}^{2} .
\end{array}
$$

We include a $5 \%$ error on the matter density. Our analysis includes the correlations between all parameters and properly accounts for possible degeneracies.

All calculations assume a normal mass hierarchy as input, whereas the fit always extends to the case of an inverted hierarchy. It is known that there are no qualitative differences in the sensitivities if an inverted hierarchy is assumed as the input; see e.g., Ref. 32]. In that case the matter enhancement moves from neutrinos to antineutrinos, but since we assume nearly symmetric neutrino and antineutrino running, the results are not affected significantly.

To allow a concise presentation of the physics results we define performance indicators for the various measurements. For $\theta_{13}$ we choose the discovery of nonzero $\sin ^{2} 2 \theta_{13}$. Here a nonzero value of $\sin ^{2} 2 \theta_{13}$ is chosen as the true value in the simulation and a fit with $\sin ^{2} 2 \theta_{13}=0$ is performed. The reach for discovering $\mathrm{CP}$ violation is computed by choosing a value for $\delta_{\mathrm{CP}}$ as input and fitting it with the two $\mathrm{CP}$ conserving values of $\delta_{\mathrm{CP}}=0, \pi$. All other parameters (including $\theta_{13}$ ) are free and the cases of an inverted mass hierarchy and $\theta_{23} \rightarrow \pi / 2-\theta_{23}$ are taken into account. For the exclusion of an inverted mass hierarchy a point in parameter space with normal mass hierarchy is chosen as the true value and the solution with the smallest $\chi^{2}$ value with inverted hierarchy has to be determined (global minimum of the $\chi^{2}$ function). The same applies to the resolution of the octant of $\theta_{23}$. Data is generated with $\theta_{23}<\pi / 4$ and the global minimum of the $\chi^{2}$ function with $\theta_{23}>\pi / 4$ has to be found.

The $\chi^{2}$ values obtained by the above procedure are converted to confidence levels by using the $\chi^{2}$ distribution for 1 degree of freedom for each of the above cases, i.e., $\sqrt{\Delta \chi^{2}}$ corresponds to the significance in Gaussian standard deviations.

\section{Results}

Our results are:

- Figure 2 shows the potential for discovering a nonzero value of $\theta_{13}$. The sensitivity does not depend strongly on the value of $\delta_{\mathrm{CP}}$. A finite value of $\theta_{13}$ can be established at $3 \sigma$ for $\sin ^{2} 2 \theta_{13}$ as low as 0.005 , irrespective of the value of $\delta_{\mathrm{CP}}$.

In what follows, we use the idea of a CP fraction, the definition of which is provided in Fig. 3 via illustration. For example, from the third panel one can see that for $75 \%$ of the values of $\delta_{\mathrm{CP}}$, the experiment can detect nonzero $\theta_{13}$ at the $3 \sigma$ C. L. if $\sin ^{2} 2 \theta_{13} \gtrsim 0.004$. The most 
optimistic case arises for a $\mathrm{CP}$ fraction of 0 since the true value of the $\mathrm{CP}$ phase is the one for which the sensitivity is maximum.

- From Fig. 4, it is evident that the baseline is not crucial in determining the ability to confirm if $\theta_{13}$ is nonzero. This independence stems from the fact that with increasing baseline $L$ the signal event rates do not drop as $L^{-2}$ but much more slowly due to enhancement by matter effects. However, the background drops faster than $L^{-2}$ since the oscillation with the solar mass splitting reduces the beam intrinsic background. This can be seen from Fig. 1] As a result, the ratio of signal to the square root of the background is approximately constant.

- In Fig. 5 we address the potential for discovering a normal mass hierarchy with a baseline of $1300 \mathrm{~km}$. A normal hierarchy can be confirmed independently of $\delta_{C P}$ at $>3 \sigma$ so long as $\sin ^{2} 2 \theta_{13}$ is larger than about 0.01 . For the inverted hierarchy the result is approximately the same because of the nearly symmetric $\nu$ and $\bar{\nu}$ running.

- Figure 6 confirms the expectation that a longer baseline improves the sensitivity to the mass hierarchy since the matter effects increase with baseline. Note that an experiment with baseline below $1000 \mathrm{~km}$ has poor sensitivity to the mass hierarchy. However, the sensitivity (approximately) plateaus for baselines above $1500 \mathrm{~km}$. As we see below, the optimal baseline is determined primarily by the discovery reach for $\mathrm{CP}$ violation.

- $\mathrm{CP}$ violation in the neutrino sector will be discovered if $\delta_{\mathrm{CP}}=0$ and $\pi$ can be excluded. Figure [7shows the sensitivity for this measurement at two baselines, $730 \mathrm{~km}$ (left-hand panel) and $1300 \mathrm{~km}$ (right-hand panel). At a baseline of $730 \mathrm{~km}$ the mass hierarchy cannot be resolved and hence the sensitivity for $\delta_{\mathrm{CP}}>0$ (for the inverted hierarchy, this occurs for $\delta_{\mathrm{CP}}<0$ ) is severely limited by so-called $\pi$-transit: for certain combinations of true $\theta_{13}$ and $\delta_{\mathrm{CP}}$ it is possible to fit the data with the wrong mass hierarchy and $\delta_{\mathrm{CP}}=\pi[31$. The existence of this solution inhibits the ability to discover $\mathrm{CP}$ violation for a range of $\delta_{\mathrm{CP}}$ in an experiment with baseline from FNAL to the Soudan mine. At a baseline of $1300 \mathrm{~km}$ the mass hierarchy is well resolved (see Fig. [5) and therefore no problems arise due to $\pi$-transit. Baselines below approximately $1000 \mathrm{~km}$ are not optimal for studying CP violation. However, also at longer baselines it can be seen that the sensitivity to $\mathrm{CP}$ violation is greater for $\delta_{\mathrm{CP}}<0$. This is mainly due to the somewhat lower statistical significance of the antineutrino data; in the antineutrino mode, the signal event rate is lower and the background event rate is higher.

- In Fig. 8, we show results for a CP fraction equal to 0.75 instead of for unity, since it is impossible for an experiment to have sensitivity to $\mathrm{CP}$ violation if $\delta_{\mathrm{CP}}=0$ or $\pi$. Note that the sensitivity decreases with baseline above $1000 \mathrm{~km}$, which is to be attributed to the competition of genuine $\mathrm{CP}$ violation and fake $\mathrm{CP}$ violation induced by matter effects. Nevertheless, the 
dependence of the sensitivity on the solar and atmospheric oscillation parameters and on the length of the baseline beyond $1000 \mathrm{~km}$ is weak and could be partly compensated by optimizing the low energy part of the spectrum. The flexibility at FNAL to vary the proton energy while keeping the total beam power constant is important for this optimization. Experiments with beams from FNAL to the Homestake mine or the Henderson mine, which have baselines of $1300 \mathrm{~km}$ and $1500 \mathrm{~km}$, respectively, have good sensitivity to $\mathrm{CP}$ violation compared to the optimal case of $1000 \mathrm{~km}$ and also have very good sensitivity to $\theta_{13}$ and the mass hierarchy. Thus, baselines around $1300-1500 \mathrm{~km}$ represent a global optimum.

- We now consider the prospect of breaking the $\left(\theta_{23}, \pi / 2-\theta_{23}\right)$ degeneracy, which is only present if the true value of $\theta_{23} \neq \pi / 4$. We display the results for two possible true values of $\theta_{23}$ in Fig. 9, To emphasize how challenging it is to break this degeneracy, we have selected values of $\theta_{23}$ that are far outside the $1 \sigma$ range in Eq. (11). The ability to exclude the wrong octant depends on the choice of the true values of $\theta_{13}$ and $\delta_{\mathrm{CP}}$. This dependence is not pronounced for most parts of the parameter space and therefore we chose to consider only the most pessimistic case, i.e. only that combination of true $\theta_{13}$ and $\delta_{\mathrm{CP}}$ which gives the lowest $\chi^{2}$ difference between the true and wrong octant. Hence, the results shown in Fig. 9 are conservative for the representative true values of $\theta_{23}$. Clearly this measurement favors baselines longer than $2000 \mathrm{~km}$. As noted earlier, such long baselines are not preferred for the detection of $\mathrm{CP}$ violation. Distinguishing the octant is a very difficult measurement which cannot be guaranteed to succeed with high confidence. If the neutrino spectrum at low energies can be enhanced, perhaps in a separate run with lower energy protons or off-axis geometry, then the signal to background ratio for this measurement could be improved.

- Finally, we assess how our results depend on exposure and on the uncertainty in the overall normalization of the background. We have not considered the systematic uncertainties in the shape of the background assuming that the background shape, which is sharply peaked at low energies, will be measured sufficiently well by a near detector, as in the MINOS experiment 33.

From Fig. 10 we see that reducing the exposure by a factor of 3, degrades the sensitivity to a nonzero $\sin ^{2} 2 \theta_{13}$ and to determine the mass hierarchy by about a factor of 2 in $\sin ^{2} 2 \theta_{13}$, while the sensitivity to $\mathrm{CP}$ violation worsens by a factor of about $2-5$ depending on the value of $\delta_{\mathrm{CP}}$; if $\delta_{\mathrm{CP}}$ has an unfavorable value, the experiment needs the highest possible exposure to maintain its ability to detect $\mathrm{CP}$ violation. Nevertheless, operation with half the total beam power, which could be achieved by the accumulator based upgrades at FNAL, is shown to have excellent sensitivity.

Similar statements can be made for the dependence on the systematic uncertainty from 
Fig. 11] Again, the sensitivity to $\mathrm{CP}$ violation puts the most stringent requirements on the experiment. The most striking conclusion is that sensitivity to the mass hierarchy is only weakly affected by the systematic uncertainty.

\section{Summary}

We have studied the measurement of $\mathrm{CP}$ violation in the neutrino sector using a powerful, but conventional neutrino beam, using a MW-class proton source located in the U.S. (either at FNAL or BNL). The beam would be delivered to a large detector with fiducial mass $\sim 300 \mathrm{kT}$ over a distance $\gtrsim 1000 \mathrm{~km}$. Such a detector could be built at a new Deep Underground Science and Engineering Laboratory (DUSEL) which is in the process of being evaluated in the U.S. The two possible sites for DUSEL at the Homestake mine in South Dakota and the Henderson mine in Colorado are at distances of $1290 \mathrm{~km}$ and $1487 \mathrm{~km}$ from FNAL, respectively (or $2540 \mathrm{~km}$ and 2770 $\mathrm{km}$ from BNL, respectively). We have calculated the scientific reach and its dependence on total exposure and systematics for a range of distances that encompass these possibilities.

The experiment is motivated by the need to have sensitivity to both the atmospheric and solar

oscillation scales (a necessary condition for observing CP violation in the 3-generation framework), and to obtain an oscillatory pattern in the energy spectrum of neutrinos. Access to multiple nodes of oscillations leads to improved sensitivity for CP violation because the CP effect grows larger for successive nodes and because parameter ambiguities can be resolved.

We have shown that the sensitivity to nonzero $\theta_{13}$ using the appearance of electron neutrinos and antineutrinos is roughly independent of baseline in the range of 1000 to $3000 \mathrm{~km}$. Also, the sensitivity to the mass hierarchy plateaus for baselines above $1500 \mathrm{~km}$. On the other hand, sensitivity to CP violation decreases with baseline above $1000 \mathrm{~km}$, but it is a weak function of the baseline beyond $1000 \mathrm{~km}$. The baselines for a beam from FNAL to Homestake or Henderson are optimal for establishing a nonzero $\theta_{13}$, the mass hierarchy and CP violation. The determination of the $\theta_{23}$ octant, however, favors baselines longer than $1500 \mathrm{~km}$. Both the $\theta_{23}$ measurement and the CP measurement could be improved by increasing the low energy part of the flux. The sensitivities for a $1300 \mathrm{~km}$ baseline are shown in Figs. 2, 5] and 7 . For the same baseline, the dependence of the sensitivities on exposure and systematic uncertainties are summarized in Figs. 10] and 111 respectively.

\section{Acknowledgments}

This research was supported by the DOE under Grants No. DE-FG02-95ER40896, DE-AC0298CH10886 and DE-FG02-04ER41308, by the NSF under CAREER Award No. PHY-0544278 and Grant No. EPS-0236913, by the State of Kansas through the Kansas Technology Enterprise 
Corporation, and by the KU General Research Fund Program. Computations were performed on facilities supported by the NSF under Grants No. EIA-032078 (GLOW), PHY-0516857 (CMS Research Program subcontract from UCLA), and PHY-0533280 (DISUN), and by the University of Wisconsin Graduate School/Wisconsin Alumni Research Foundation.

\section{References}

[1] For a review see, V. Barger, D. Marfatia and K. Whisnant, Int. J. Mod. Phys. E 12, 569 (2003) arXiv:hep-ph/0308123.

[2] M. Ishitsuka [Super-Kamiokande Collaboration], arXiv:hep-ex/0406076

[3] K. Eguchi et al. [KamLAND Collaboration], Phys. Rev. Lett. 90, 021802 (2003) arXiv:hep-ex/0212021; T. Araki et al., arXiv:hep-ex/0406035

[4] M. Apollonio et al. [CHOOZ Collaboration] Eur. Phys. J. C 27, 331 (2003) arXiv:hep-ex/0301017.

[5] Y. Itow et al., arXiv:hep-ex/0106019.

[6] D. S. Ayres et al. [NOvA Collaboration], arXiv:hep-ex/0503053.

[7] D. Beavis et al., arXiv:hep-ex/0205040

[8] M. Diwan et al., Phys. Rev. D 68, 012002 (2003) arXiv:hep-ph/0303081.

[9] D. Beavis et al., E889 Collaboration, BNL preprint BNL-52459, April 1995.

[10] V. Barger, D. Marfatia and K. Whisnant, Phys. Rev. D 65, 073023 (2002) arXiv:hep-ph/0112119.

[11] J. Burguet-Castell, M. B. Gavela, J. J. Gomez-Cadenas, P. Hernandez and O. Mena, Nucl. Phys. B 608, 301 (2001) arXiv:hep-ph/0103258; H. Minakata and H. Nunokawa, JHEP 0110, 001 (2001) arXiv:hep-ph/0108085; G. L. Fogli and E. Lisi, Phys. Rev. D 54, 3667 (1996) arXiv:hep-ph/9604415.

[12] V. Barger, D. Marfatia and K. Whisnant, Phys. Rev. D 66, 053007 (2002) arXiv:hep-ph/0206038; Phys. Lett. B 560, 75 (2003) arXiv:hep-ph/0210428; in Proc. of the APS/DPF/DPB Summer Study on the Future of Particle Physics (Snowmass 2001) ed. N. Graf, eConf C010630, E102 (2001) arXiv:hep-ph/0108090; P. Huber, M. Lindner and W. Winter, Nucl. Phys. B 654, 3 (2003) arXiv:hep-ph/0211300; O. Mena Requejo, S. Palomares-Ruiz and S. Pascoli, Phys. Rev. D 72, 053002 (2005) arXiv:hep-ph/0504015; Phys. Rev. D 73, 073007 (2006) arXiv:hep-ph/0510182; O. Mena and S. J. Parke, Phys. 
Rev. D 72, 053003 (2005) arXiv:hep-ph/0505202; M. Ishitsuka, T. Kajita, H. Minakata and H. Nunokawa, Phys. Rev. D 72, 033003 (2005) arXiv:hep-ph/0504026.

[13] M. V. Diwan et al., arXiv:hep-ex/0608023.

[14] R. J. Wilkes, arXiv:hep-ex/0507097; C. K. Jung, AIP Conf. Proc. 533, 29 (2000) arXiv:hep-ex/0005046.

[15] D. B. Cline, F. Raffaelli and F. Sergiampietri, arXiv:astro-ph/0604548 A. Ereditato and A. Rubbia, Nucl. Phys. Proc. Suppl. 155, 233 (2006) arXiv:hep-ph/0510131; A. Rubbia, arXiv:hep-ph/0402110 D. B. Cline, F. Sergiampietri, J. G. Learned and K. McDonald, Nucl. Instrum. Meth. A 503, 136 (2003) arXiv:astro-ph/0105442.

[16] See http://www.dusel.org/ and Neutrinos and Beyond: New Windows on Nature, Neutrino Facilities Assesment Committee, National Research Council, (2003), ISBN-0-309-087 16-3, http://www.nap.edu/catalog/10583.html.

[17] M. V. Diwan, arXiv:hep-ex/0407047.

[18] C. Yanagisawa, C. Jung, P. T. Lee, and B. Viren, Preprint submitted to The U.S. Long Baseline Neutrino Experiment Study, July 2006, http://nwg.phy.bnl.gov/ diwan/nwg/fnalbnl/docs/numunuePREBWv3.pdf

[19] R. Alber, et al., Proton Driver Study Group FNAL-TM-2136, FNAL-TM-2169. http://www-bd.fnal.gov/pdriver/

[20] R. Zwaska (For SNuMI planning group), July 17, 2006. FNAL-Beams-DOC-2393.

[21] J. Alessi et al., AGS Super Neutrino Beam Facility, Accelerator and Target System Design, BNL-71228-2003-IR. April 15, 2003. http://nwg.phy.bnl.gov/

[22] D. Mcginnis, Beams Document 1782-v7, FNAL, 2005.

[23] S. A. Kahn and M. Diwan, PAC-2005-RPPT059 Prepared for Particle Accelerator Conference (PAC 05), Knoxville, Tennessee, 16-20 May 2005.

[24] M. Bishai, J. Heim, C. Lewis, A. Marino, B. Viren and F. Yumicea, Preprint submitted to the U.S. Long Baseline Neutrino Experiment Study, July 2006, BNL-76997-2006IR.

[25] Y. Fukuda et al., Nucl. Instrum. Meth. A 501, 418 (2003).

[26] M. H. Ahn [K2K Collaboration], arXiv:hep-ex/0606032.

[27] S. L. Adler, Annals Phys. 50, 189 (1968); D. Rein and L. M. Sehgal, Annals Phys. 133, 79 (1981). 
[28] M. Diwan et al., arXiv:hep-ex/0211001

[29] P. Huber, M. Lindner and W. Winter, Comput. Phys. Commun. 167, 195 (2005) arXiv:hep-ph/0407333.

[30] S. Eidelman et al. [Particle Data Group], Phys. Lett. B 592, 1 (2004).

[31] P. Huber, M. Lindner and W. Winter, Nucl. Phys. B 645, 3 (2002) arXiv:hep-ph/0204352.

[32] P. Huber, M. Lindner, M. Rolinec and W. Winter, Phys. Rev. D 73, 053002 (2006) arXiv:hep-ph/0506237.

[33] A. Marchionni [MINOS Collaboration], Nucl. Phys. Proc. Suppl. 155, 162 (2006). 

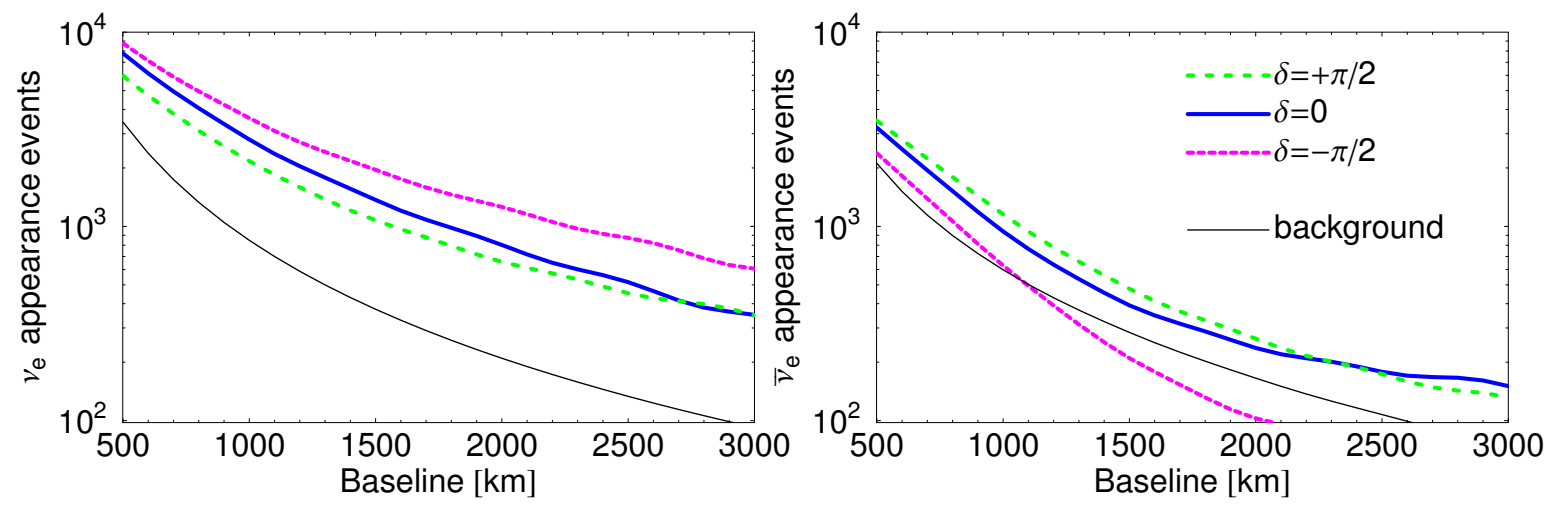

Figure 1: Event rates for neutrinos (left-hand panel) and antineutrinos (right-hand panel) as a function of baseline for $\sin ^{2} 2 \theta_{13}=0.1$ and a normal hierarchy. The bold lines show the signal for various choices of $\delta_{\mathrm{CP}}$. The thin line shows the total background, which includes background from beam contamination and neutral current events.

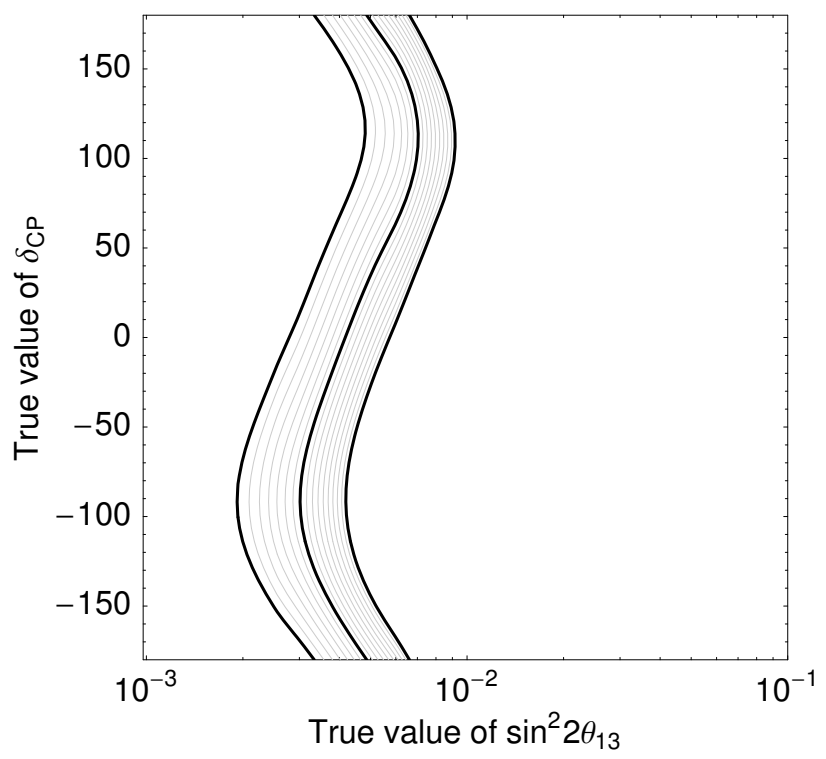

Figure 2: Discovery potential for $\sin ^{2} 2 \theta_{13} \neq 0$ at a baseline of $1300 \mathrm{~km}$. The bold iso- $\chi^{2}$ lines are $3,4,5 \sigma$ (from left to right) and the light lines show an increase of $\chi^{2}$ by 1 . For all points to the right of the rightmost bold line, a nonzero value of $\sin ^{2} 2 \theta_{13}$ can be established with at least $5 \sigma$ significance. 

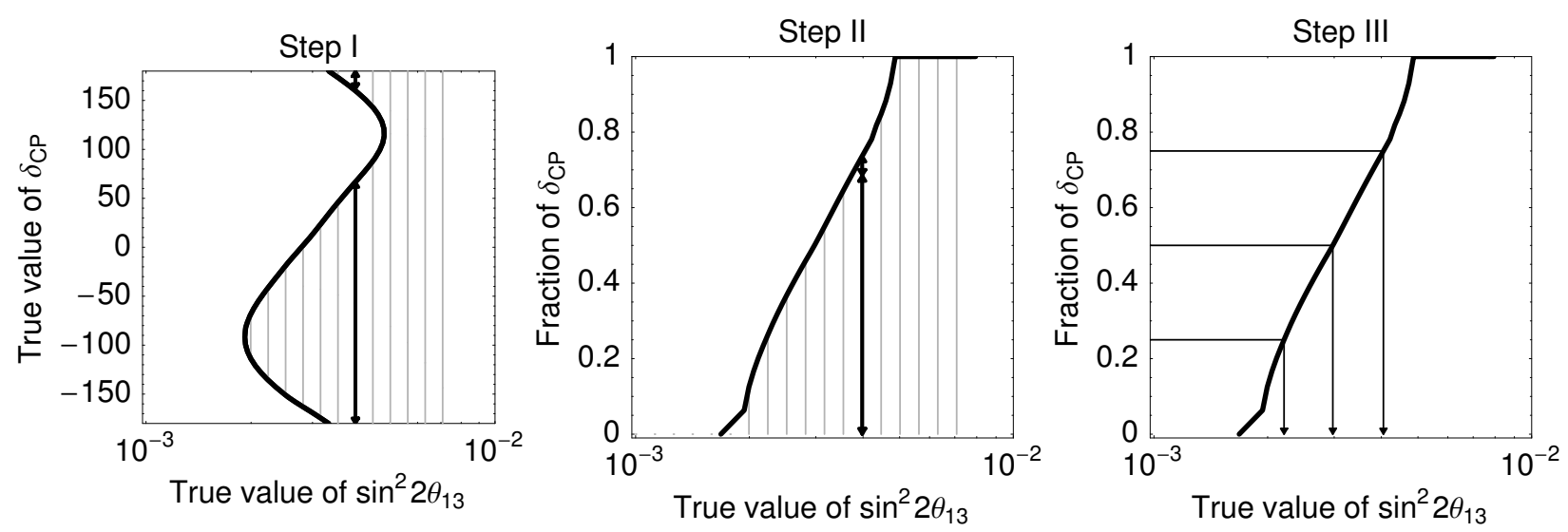

Figure 3: Schematic definition of the CP fraction. First, for each value of $\sin ^{2} 2 \theta_{13}$, the result in the $\theta_{13}-\delta_{\mathrm{CP}}$ plane is used to determine the fraction of all CP phases for which there is sensitivity at the given confidence level (black arrows). Here we have taken the $3 \sigma$ curve of Fig. 2] Repeating this for all possible values of $\sin ^{2} 2 \theta_{13}$ yields the figure shown in the middle panel. In the last step one chooses a set of values for the CP fraction and translates them into values of $\sin ^{2} 2 \theta_{13}$ by using the result from the second step.

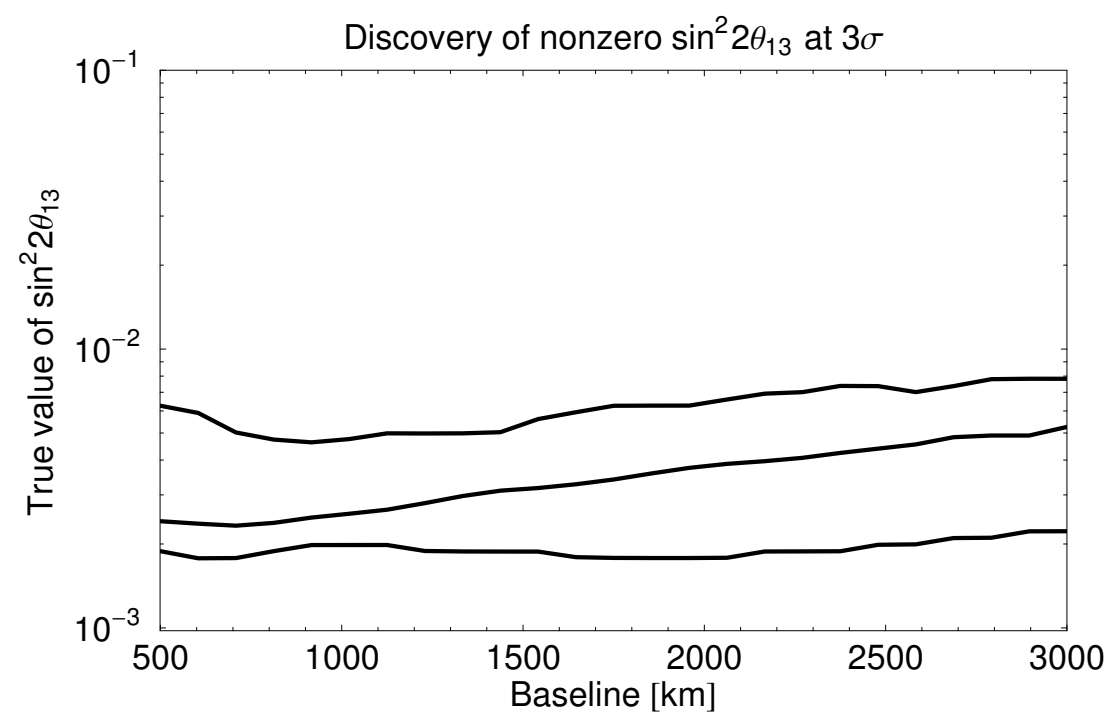

Figure 4: Discovery reach for $\sin ^{2} 2 \theta_{13} \neq 0$ at $3 \sigma$ for CP fractions 0 (lowermost line, best case), 0.5 (middle line) and 1 (uppermost line, worst case) as a function of the baseline. The detector mass, beam power and exposure are kept the same for all baselines. 


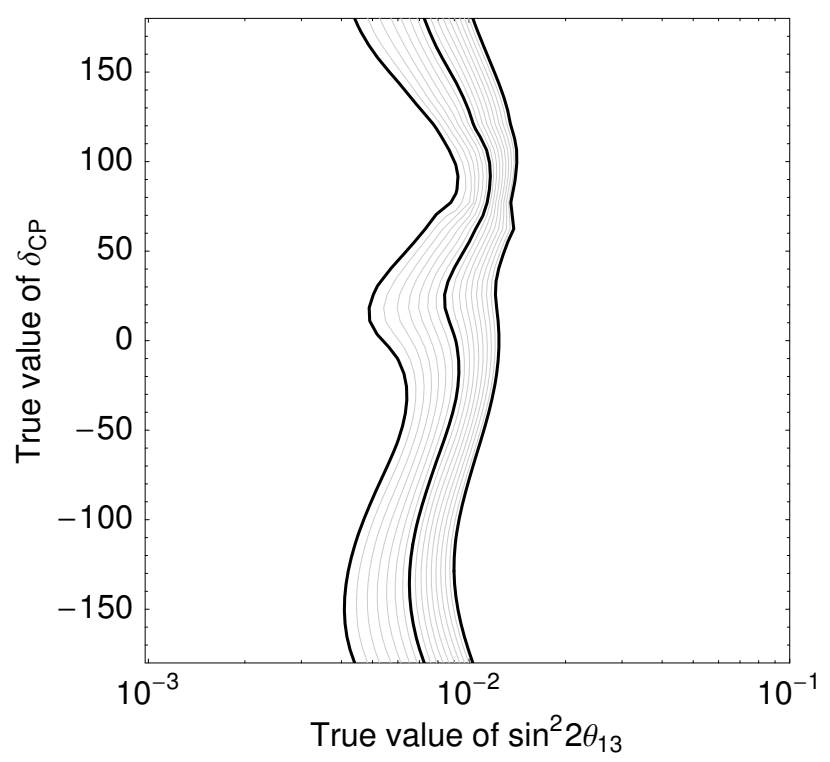

Figure 5: Discovery potential for a normal mass hierarchy at a baseline of $1300 \mathrm{~km}$. The bold iso- $\chi^{2}$ lines are $3,4,5 \sigma$ (from left to right) and the light lines show an increase of $\chi^{2}$ by 1 . For the inverted hierarchy the results are approximately the same because of the approximately symmetric $\nu$ and $\bar{\nu}$ running.

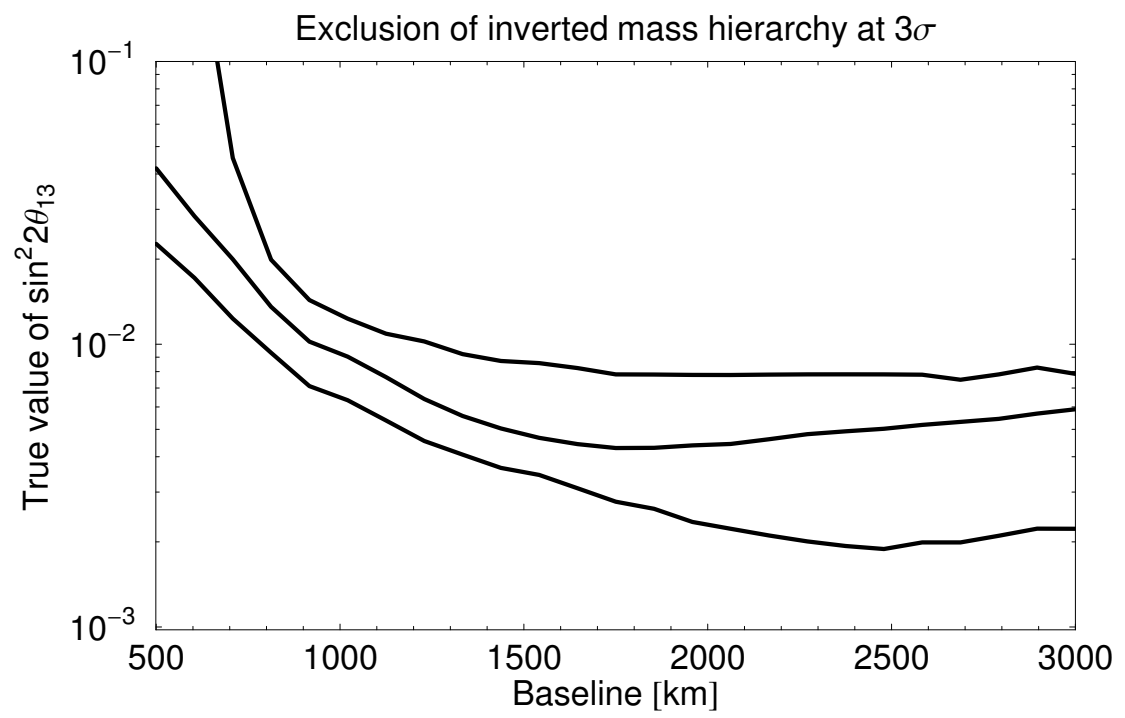

Figure 6: Discovery reach for a normal mass hierarchy at $3 \sigma$ for $\mathrm{CP}$ fractions 0 (lowermost line, best case), 0.5 (middle line) and 1 (uppermost line, worst case) as a function of the baseline. The detector mass, beam power and exposure are kept the same for all baselines. 

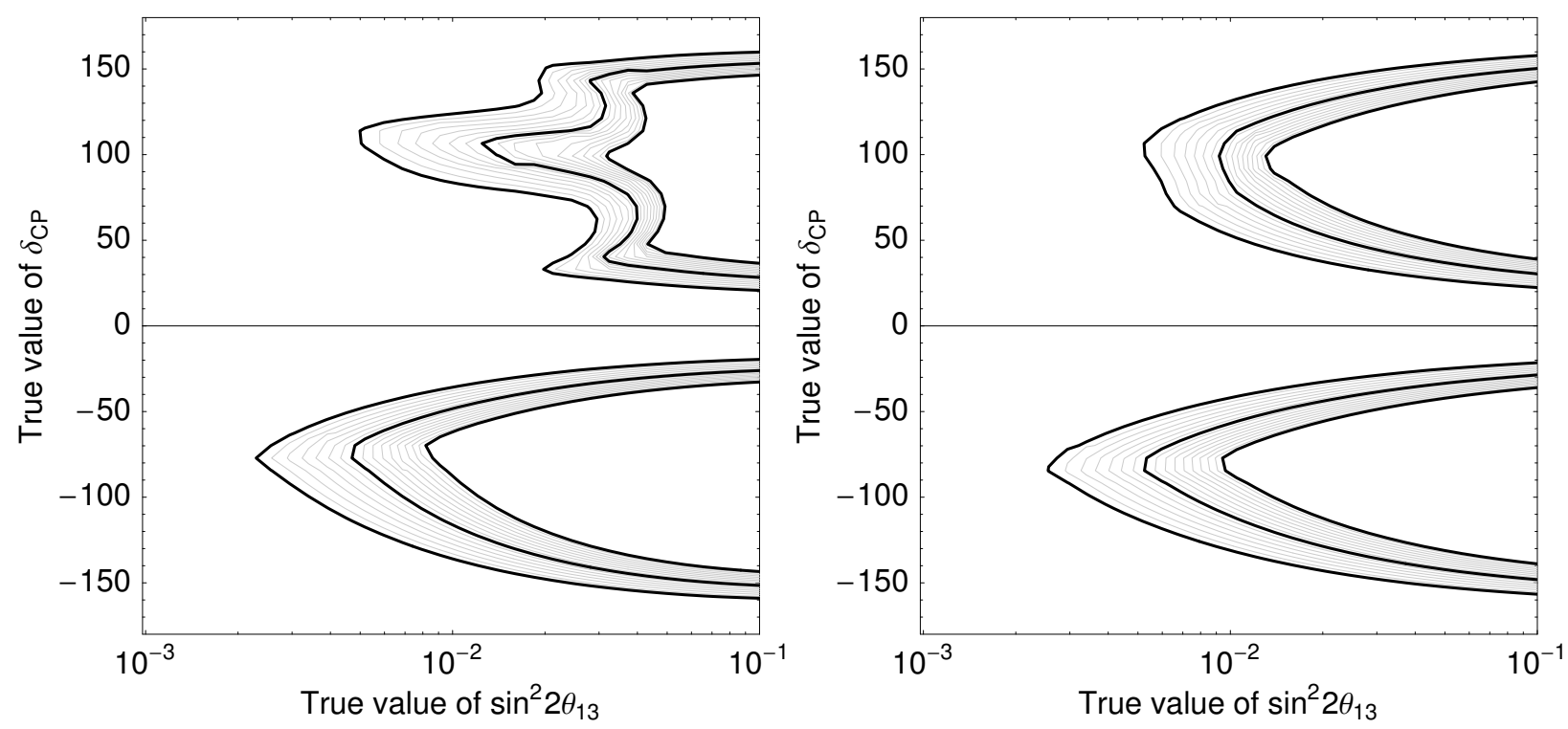

Figure 7: Discovery potential for CP violation at baselines of $730 \mathrm{~km}$ (left-hand panel) and $1300 \mathrm{~km}$ (right-hand panel). The bold iso- $\chi^{2}$ lines are 3,4,5 $\sigma$ (from left to right) and the light lines show an increase of $\chi^{2}$ by 1 . For all points to the right of the rightmost bold line, CP violation can be established with at least $5 \sigma$ significance.

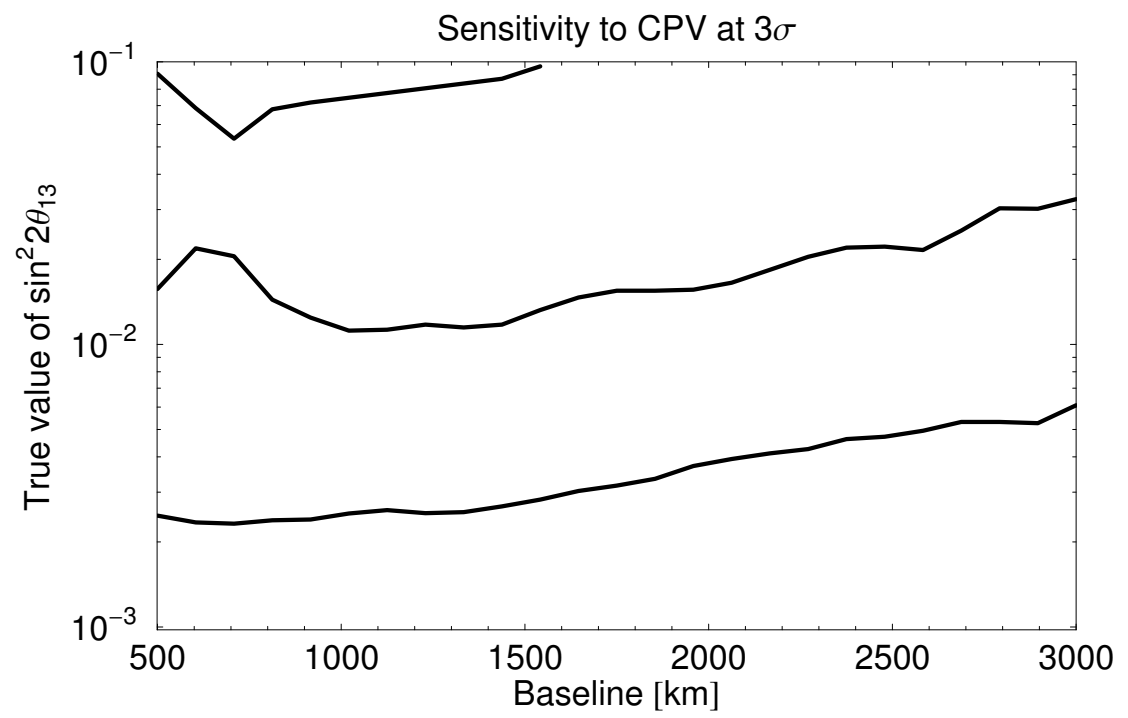

Figure 8: Discovery reach for $\mathrm{CP}$ violation at $3 \sigma$ for $\mathrm{CP}$ fractions 0 (lowermost line, best case), 0.5 (middle line) and 0.75 (uppermost line) as a function of the baseline. The detector mass, beam power and exposure are kept the same for all baselines. 


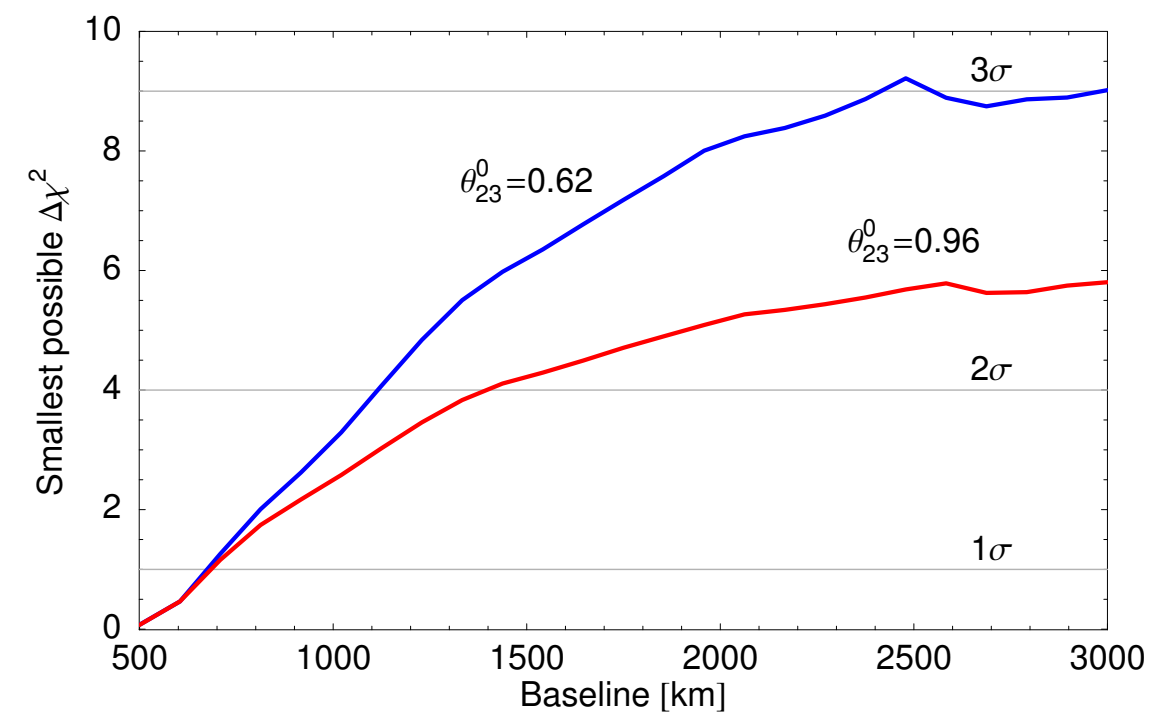

Figure 9: Discovery reach for the octant of $\theta_{23}$. Only the most conservative case with respect to the true values of $\theta_{13}$ and $\delta_{\mathrm{CP}}$ is considered. The $\chi^{2}$ difference between the true and wrong octant is shown as a function of the baseline for two representative true values of $\theta_{23}$ that are far outside the $1 \sigma$ range in Eq. (10) (so as to emphasize how challenging this measurement is). The detector mass, beam power and exposure are kept the same for all baselines.
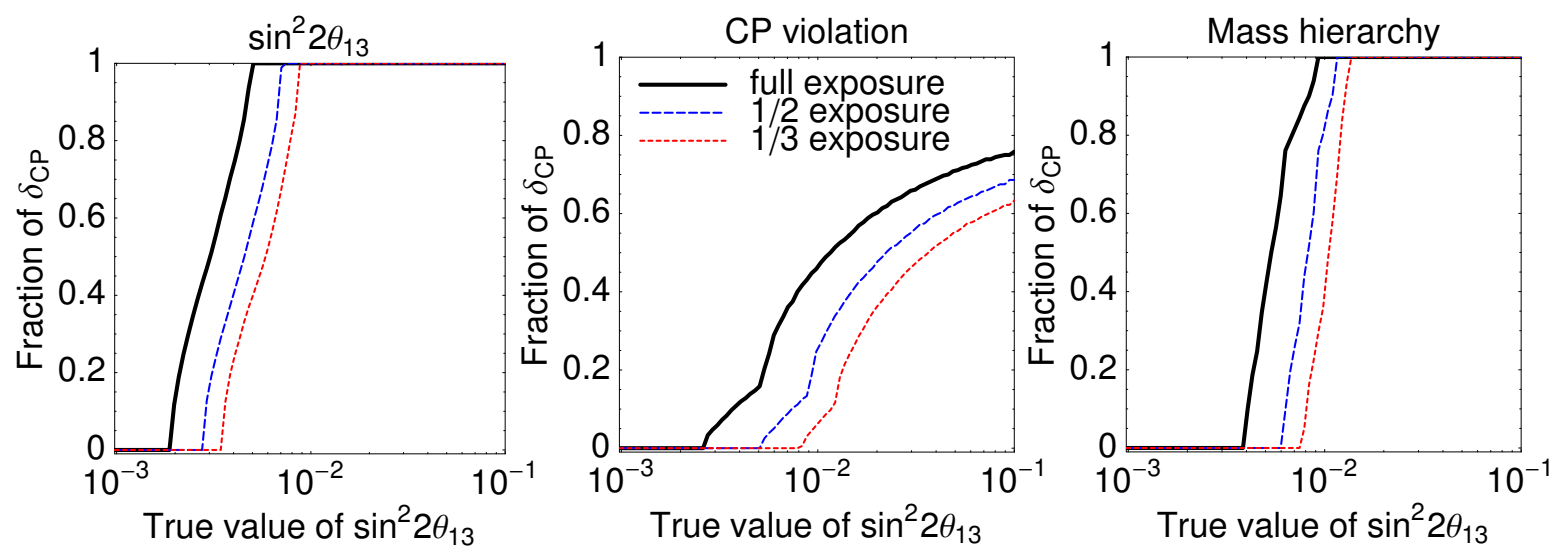

Figure 10: Dependence of the $3 \sigma$ sensitivities on exposure for an experiment with $1300 \mathrm{~km}$ baseline. The two cases considered are $1 / 2$ and $1 / 3$ of the (full) exposure we have used throughout. 

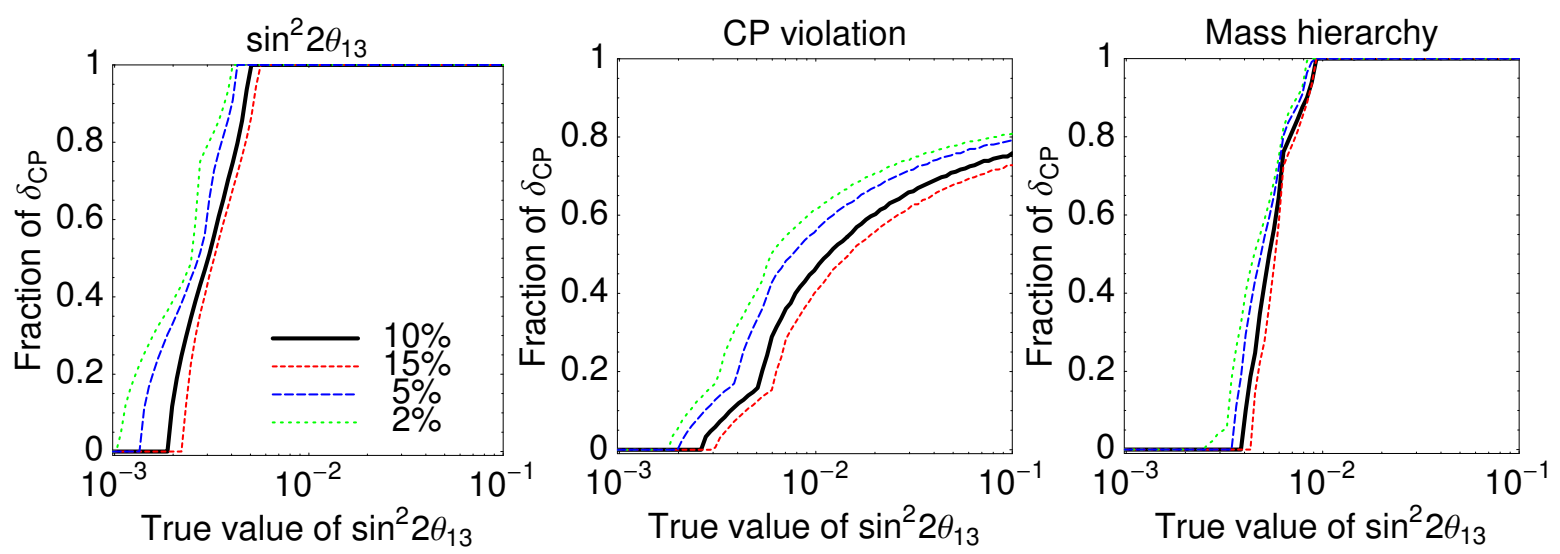

Figure 11: Dependence of the $3 \sigma$ sensitivities on the uncertainty in the overall normalization of the background for an experiment with a $1300 \mathrm{~km}$ baseline. We have adopted a $10 \%$ uncertainty throughout. 